\title{
Halmstad University Post-Print
}

\section{Incremental redundancy deadline dependent coding for efficient wireless real-time communications}

Elisabeth Uhlemann and Lars K. Rasmussen

N.B.: When citing this work, cite the original article.

(C)2005 IEEE. Personal use of this material is permitted. However, permission to reprint/republish this material for advertising or promotional purposes or for creating new collective works for resale or redistribution to servers or lists, or to reuse any copyrighted component of this work in other works must be obtained from the IEEE.

Uhlemann E, Rasmussen L. Incremental redundancy deadline dependent coding for efficient wireless real-time communications. In: IEEE Conference on Emerging Technologies and Factory Automation, 2005. Piscataway, N.J.: IEEE; 2005. p. 417-424.

DOI: http://dx.doi.org/10.1109/ETFA.2005.1612708

Copyright: IEEE

Post-Print available at: Halmstad University DiVA

http://urn.kb.se/resolve?urn=urn:nbn:se: hh:diva-378 


\title{
Incremental Redundancy Deadline Dependent Coding for Efficient Wireless Real-Time Communications
}

\author{
Elisabeth Uhlemann $^{\dagger, \star}$ and Lars K. Rasmussen ${ }^{\S}$ \\ ${ }^{\dagger}$ Centre for Research on Embedded *Volvo Technology Corporation \\ ${ }^{\S}$ Inst. for Telecommun. Research \\ Systems, Halmstad University Transport and Telematics Services \\ Box 823, 30118 Halmstad, Sweden Dept. 6600, M1:6, Götaverksg. 10 \\ University of South Australia \\ Elisabeth.Uhlemann@ide.hh.se \\ SE-405 08 Göteborg, Sweden \\ Mawson Lakes SA 5095, Australia \\ Lars.Rasmussen@unisa.edu.au
}

\begin{abstract}
The concept of deadline dependent coding (DDC) has been demonstrated as a promising design approach for efficient and reliable real-time communications over wireless channels. The main idea behind the concept of $D D C$ is to make all components of the communication protocol deadline dependent, tailoring the channel code and the retransmission protocol to the specific real-time constraints. The DDC framework allows critical reliability and timing constraints to be readily evaluated as a function of available system resources. Concatenated coding and iterative decoding within retransmission protocols enables additional flexibility and new adaptive design options. Here, we introduce an incremental redundancy retransmission scheme in conjunction with concatenated coding and show that this further improves the DDC scheme.
\end{abstract}

\section{Introduction}

Most embedded systems are expected to provide increasingly more complex and safety-critical services. Cooperation and communication between separated embedded systems typically found in a factory automation environment enables enhanced applications with strict real-time and reliability requirements. Traditionally, communication in such production environments is based on conventional wireline techniques. Wireless communications, however, provides a new level of design freedom and flexibility. Looking beyond simple replacement of existing wireline solutions, entirely new applications enabled exclusively by wireless communi-

This work was partly funded by the Knowledge Foundation, the national Swedish Real-Time research initiative ARTES supported by the Swedish Foundation for Strategic Research and by Personal Computing and Communication (PCC ++ ) under Grant PCC-0201-09. L. K. Rasmussen is supported by the Australian Government under ARC Grant DP0558861 and by the Swedish Research Council through Chalmers University of Technology under grants 621-2001-2976 and 723-2002-4533. cations between system components can also be envisioned. This vision is supported by the ongoing wireless revolution when considering the trend towards cooperative embedded systems in all kinds of home equipment, automotive components, entertainment devices and logistics services.

Even though wireless communication offers considerable advantages for industry automation applications, the hostile wireless transmission environment has limited its extensive use in systems with real-time constraints. In a typical wireless communication system, the channel conditions vary with time [1]. The inherent consequence of this is a relatively high average error rate, making the wireless channel significantly less reliable in comparison to copper wire and optical channels. Techniques for providing strict reliability and deadline guarantees for transmission over wireless channels must therefore be developed.

The Deadline Dependent Coding (DDC) scheme presented by the authors in [2] and formalized in [3] addresses these issues. The purpose of DDC is to improve the reliability of real-time communication over a wireless channel by means of specifically tailored channel coding. This is accomplished using forward error control codes in conjunction with a retransmission scheme, a socalled automatic repeat request (ARQ) scheme. The Reed-Solomon codes used in [2,3] were replaced by powerful concatenated codes with iterative decoding in [4]. Iterative techniques provide an entirely new level of performance and flexibility for DDC systems, making the approach very attractive. These schemes are closely related to turbo coding, [5], which provides virtually optimal use of the fundamental channel resources. Using such strategies in a retransmission scheme also elevates the corresponding performance to levels close to fundamental limits.

In this paper we keep the concatenated codes, but replace the block repetition hybrid ARQ protocol used in [2-4] with a more efficient incremental redundancy (IR) hybrid ARQ (IR-HARQ) scheme. IR is tractable since no bits are transmitted more than once and hence repetition of previously transmitted bits is avoided. IR was first suggested in [6] and is considered here in the DDC 
framework. The redundant bits are ordered and partitioned for each transmission with the aim of obtaining as high reliability as fast as possible, using a minimum of resources.

The paper is organized as follows. Section 2 reviews the idea and benefits of DDC for wireless real-time communications. In section 3 the purpose of using concatenated coding for DDC is discussed, while section 4 describes the concept of IR in hybrid ARQ protocols. In section 5 the effect of correctly ordering the redundancy before transmission is presented and its impact on the DDC scheme illustrated. Further, in section 6 the benefits of choosing correct packet lengths for each retransmission is discussed and the effect on DDC is demonstrated. Finally, section 7 contains our conclusions.

\section{Deadline dependent coding}

The objective of DDC is to enable efficient real-time communications in relevant applications for future mobile wireless communication networks. Development of specifically tailored channel codes makes the data link layer and parts of the network layer protocols suitable for reliable and efficient wireless real-time communication.

Network protocols such as IP [7] have traditionally been designed for a best-effort approach regarding both timeliness and information reliability. The protocol suite TCP/IP is a joint transport and network layer protocol suite that provides reliable communication through error detection and retransmission strategies, but offers only best-effort timeliness. Recently, quality of service (QoS) implementations extending into new IP standards have made it possible to introduce priority algorithms, e.g. IPv6 [8]. Also the use of the UDP protocol in place of TCP has made it possible to provide real-time multimedia communication at best-effort information reliability. These extensions are based on modifications to protocols originally designed for different purposes, which means that most existing protocol standards can only guarantee timeliness or information reliability, but not both.

Besides TCP/IP there are a number of real-time communication protocols such as [9-11] that strives to ensure delivery prior to deadlines. These protocols are best-effort schemes in terms of information reliability and consequently do not give any guarantees or explicit prediction on the probability of correct delivery. Recent protocols offering negotiable QoS in terms of timeliness requirements have been suggested in e.g., $[12,13]$. They assume, however, a reliable channel which is directly available for optical wired applications. Unfortunately, in a mobile wireless environment, the encountered communication conditions are quite challenging compared to wired communication in terms of signal degradation. The inherent error rate being significantly higher in a wireless environment has prevented extensive use of wireless communication in real-time systems with criti- cal deadlines. The concepts of channel coding and retransmission schemes must be introduced in order to provide a reliable channel.

Another difficulty with a wireless channel is its limited bandwidth. The radio spectrum is a limited natural resource, assigned according to strictly enforced rules and consequently additional bandwidth may be costly or may not exist at all. Furthermore, wireless devices are often battery powered and therefore put restrictions on the maximal computational complexity and the transmitted signal power to prolong battery life. Moreover, given that we have a limited bandwidth, liming the transmitted power also limits the inherent interference generated by other transmitters present in the local wireless multiple access system. All of this implies that the channel code and the retransmission protocol have to be carefully tailored.

The main idea behind DDC is to make each component in the communication protocol deadline dependent. In addition to being subject to a real-time deadline, the information must also be delivered correctly with high probability since information delivered before the deadline, but in error, may have severe consequences. The DDC communication protocol lets the timeliness and the reliability of delivered information constitute the QoS parameters required by the application. We thus consider the probability of correct delivery, $P_{d}$, prior to reaching the deadline $t_{D L}$. Correct delivery implies that a certain target error rate, $P_{t}$, is met. Note that the target error rate can never be equal to zero due to the presence of channel noise [1].

Using these QoS parameters it follows that a protocol layer can negotiate values of the parameters with an upper or a lower layer, thus enabling flexible admission control that provides a trade-off between the delivery time and the quality of the delivered data. The values of the QoS parameters are requested by the application using the communication system. The value of $P_{d}$ controls how reliable the transfer must be in a real-time perspective, i.e., a measure of how critical the task is, and consequently, it does not say anything about delivery of correct information after the designated deadline. The negotiation about the value of $t_{D L}$ reflects how soft the real-time constraints on the deadline are.

One of the objectives of the wireless real-time communication protocol is to maximize the probability that the communication system will be able to accept the transmission request with the required values of the QoS parameters. Besides maximizing the probability of delivering the necessary information before a given deadline, the protocol should also attempt to minimize the required bandwidth, the transmitted energy and the average time required to successfully deliver the information. The values of the QoS parameters are transformed into actions to be taken by the link layer protocol in terms of coding and retransmission strategies specifically required when using an unreliable channel. Consequently, 
the QoS parameters $t_{D L}$ and $P_{d}$ are mapped onto a retransmission protocol which plays the role of maximizing the probability of correct delivery before the deadline using a minimum of resources. The DDC protocol thus performs a series of transmissions triggered by the retransmission protocol, providing increasingly more information for decoding the closer we come to the deadline.

\section{Concatenated codes}

The channel capacity formulated by Shannon [14] incorporates into one composite parameter the effects of channel parameters such as thermal noise, constrained bandwidth, and limited signal power. The significance of the channel capacity is that as long as the communication rate is kept below the channel capacity, an arbitrarily low error rate can in principle be obtained if infinitely long codes are used. Note that the term "long code" used here does not refer to the length of the information frame, but rather the length of the codeword, or the length of the code memory. Decoding complexity, however, generally increases exponentially with code length and hence may prohibit the use of codes beyond a certain length. At the same time as a long code will improve the performance in terms of lowering the bit error rate (BER), it will also require more resources in terms of more bandwidth, more energy and above all, in this context, more time to transmit and decode. When a real-time communication system is considered, time is a limited resource and hence we are not only concerned with limiting the length of the code in order to limit the decoding complexity but also to limit the overall communication time.

The channel capacity unfortunately only states what communication rate is theoretically possible to achieve, but it does not say what codes to use in order to achieve an arbitrary low BER for a particular rate. Therefore, there has been a gap between the theoretical limit and the achievable communication rates obtained using codes of a manageable decoding complexity. Concatenated codes using iterative decoding is a relatively new way of providing long codes capable of virtually closing the performance gap while maintaining manageable decoding complexity. A turbo code is basically a concatenated system of two simple component codes connected through an interleaver in order to create a very long code and also a very strong code $[5,15]$. Optimal decoding of such a system is intractable due to the length of the code as determined by the size of the interleaver. However, the attractive characteristic of a concatenated system is that iterative a posteriori probability (APP) decoding based on exchanging soft reliability information between the code components provides a low complexity suboptimal decoding algorithm. This implies that each component decoder is used several times in the decoding process, usually once for each iteration. Hence, the soft reliability information exchanged between the component decoders is iteratively refined and the interleaver is used as an integrated part of the code. The overall decoding complexity of the iterative decoding algorithm for a concatenated code is lower than that required for a single code of the corresponding performance. The lower complexity is achieved by decoding each component code separately. The low-complexity decoders for the simple component codes are used and iteratively reused several times, instead of using one highly complex optimal decoder for the full code.

In general, concatenated coding provides longer codes that yield significant performance improvements at reasonable complexity investments. Given certain conditions, the iterative decoding algorithm performs close to the fundamental Shannon capacity [5]. The ideas behind DDC have been extended by the authors in [4] to include the principles of concatenated coding and powerful iterative decoding.

\section{Incremental redundancy}

Whenever a feedback channel is available, an ARQ scheme [16] can be used. This implies that when a packet arrives, the receiver may choose not to accept it, but instead request a retransmission through the feedback channel. This request can be done either by sending a negative acknowledgement or simply by not sending any acknowledgement. To determine whether or not a retransmission request should be generated, the receiver checks the quality or the reliability of the received packet. This two-way communication usually goes on until the receiver obtains a packet that is considered sufficiently reliable.

A hybrid ARQ (HARQ) scheme [17] uses an error control code in conjunction with the retransmission scheme. Consequently, the received packet is first decoded and a retransmission is requested only if the quality of the decoding decision is too low, i.e. if the decoded sequence is below a certain reliability threshold. There are different methods of determining whether a decoding decision is sufficiently reliable and hence different criteria for requesting a retransmission. The choice of method significantly affects the character of the retransmission scheme.

There are also different ways of handling packets responsible for causing a retransmission. These "erroneous" packets can either be dropped or they can be combined with one or more retransmitted packets. This procedure of using the information in previously received packets is termed packet combining and was first suggested in [18]. There are two different ways of using previously received packets in order to improve performance, i.e. two different packet combining techniques, diversity combining, [18] and code combining, e.g., [19]. Diversity combining is typically implemented before the decoder on a symbol-by-symbol (or bit-by- 
bit) basis. Individual symbols from multiple identical copies of a packet are combined to create a single packet of the same length but with more reliable constituent symbols. In contrast, a code combining scheme concatenates several packets pertaining to the same frame on a packet-by-packet basis to form a codeword of lower rate, i.e., containing more redundant bits.

Further, there are different types of HARQ schemes which basically are defined by their respective packet combining method. HARQ type-I, [17] uses no packet combining and hence erroneous packets are simply dropped. HARQ type-II, [20] uses code combining so that increasingly longer codewords with lower and lower code rate are formed for each retransmission. Finally, HARQ type-III, [21] uses diversity combining implying that identical packets are retransmitted and combined to form a new more reliable packet with maintained code rate. HARQ type-III is essentially packet repetition and consequently just a variant of an HARQ type-II scheme using a simple repetition code.

Previous versions of the DDC protocol [2-4] have used HARQ type-III. In this work an HARQ scheme of type-II is used, i.e., a code combining scheme using IR. IR implies that the HARQ scheme responds to a retransmission request by transmitting increasingly more redundancy. This redundancy is then appended to the previously received packet, thus lowering the code rate This way more advanced codes than repetition codes can be created by the retransmissions. IR is usually accomplished by so-called rate compatible punctured codes Puncturing a code implies that certain code bits are removed before transmission so that the overall code rate is increased. The receiver needs to know the so-called puncturing pattern, so that erasures can be inserted instead of the punctured bits before decoding starts. The punctured bits can then later be sent in a retransmission and thus bit by bit complete the lower rate codeword in the receiver. A family of rate compatible codes implies that all code bits belonging to a particular code in the family also belongs to all other codes with a lower code rate in that family. The transmitter and receiver only need to share a puncturing table to determine which code bits are to be transmitted next, and the receiver simply inserts erasures for all redundant bits that have not yet been received. That way, starting with a high rate code the transmitter only needs to transmit complementary redundant bits to get to the next lowest rate code, and hence incremental redundancy can be accommodated.

\section{IR transmission order}

Since no repetition of previously transmitted parity bits is made in an IR scheme, the following notation is adopted. A data frame is a sequence of $k$ information bits passed through an encoder with code rate $k / n$, and thus generating a codeword of length $n$ with $n-k$ parity bits or redundant bits. This code is termed the mother code.
The codeword is then partitioned into $M \geq 1$ IR-blocks as follows. For $i=1,2, \ldots, M$, let $f_{i}$ denote transmission $i$ and let $c_{i}$ denote the number of redundant bits included in $f_{i}$, such that $\sum_{i=1}^{M} c_{i}=n-k$ and $0 \leq c_{i} \leq n-k$. Note that $f_{1}$ includes the $k$ information bits as well as $c_{1}$ redundant bits, whereas for $i>1, f_{i}$ includes only the redundant bits $c_{i}$.

When $f_{i}$ for $i<M$, is received, the decoder inserts the newly arrived redundant bits at their respective places and consequently decodes a codeword of length $n_{i}=k+\sum_{j=1}^{i} c_{j}$. If the reliability of the decoding decision is too low, transmission $f_{i+1}$ is requested. The decoder output is delivered to the user application as soon as decoding is successful or whenever the maximum number of packets $M$ has been received. The target frame error rate $P_{t}$ can thus be obtained by proper design of the mother code.

As an example mother code we have chosen a parallel concatenated single parity check (SPC) code. Our SPC code takes a block of seven information bits and adds a single parity bit to it. We use three SPC codes concatenated in parallel and separated by two interleavers of size $7 \cdot 49=343$. Each encoder thus adds 49 redundant bits to every data frame of size 343. Consequently, $k=343$ and $n-k=3 \cdot 49=147$. We assume that this mother code will provide the required level of $P_{t}$.

Further, we assume that $t_{D L}$ and the round trip delay allows a maximum of $M=7$ transmissions or partitions of the mother codeword. For simplicity we will choose the packet sizes according to Table 1 . This implies that 24 of the 147 available parity bits should be included in the first retransmission should it become necessary.

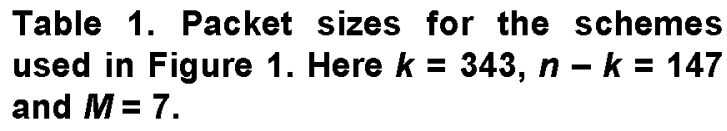

\begin{tabular}{|c|c|c|c|c|c|c|c|}
\hline & $f_{1}$ & $f_{2}$ & $f_{3}$ & $f_{4}$ & $f_{5}$ & $f_{6}$ & $f_{7}$ \\
\hline$c_{i}$ & 0 & 24 & 25 & 24 & 25 & 24 & 25 \\
\hline$n_{i}$ & 343 & 367 & 392 & 416 & 441 & 465 & 490 \\
\hline
\end{tabular}

An interesting question is now whether it matters how these 24 redundant bits are chosen, i.e., we are interested in knowing whether one particular puncturing pattern leads to successful decoding faster than another. Due to the regular structure of the chosen mother code, there are two cases that are particularly interesting: choosing all 24 bits among the parity bits generated by the same encoder or choosing them randomly from all available encoders. We term the first option dimension-wise puncturing and the latter random puncturing.

By means of Monte-Carlo simulations we compare two schemes using random puncturing and dimensionwise puncturing respectively. Both schemes use the same mother code and packet lengths according to Table 1 . The codewords are transmitted using binary phase shift 


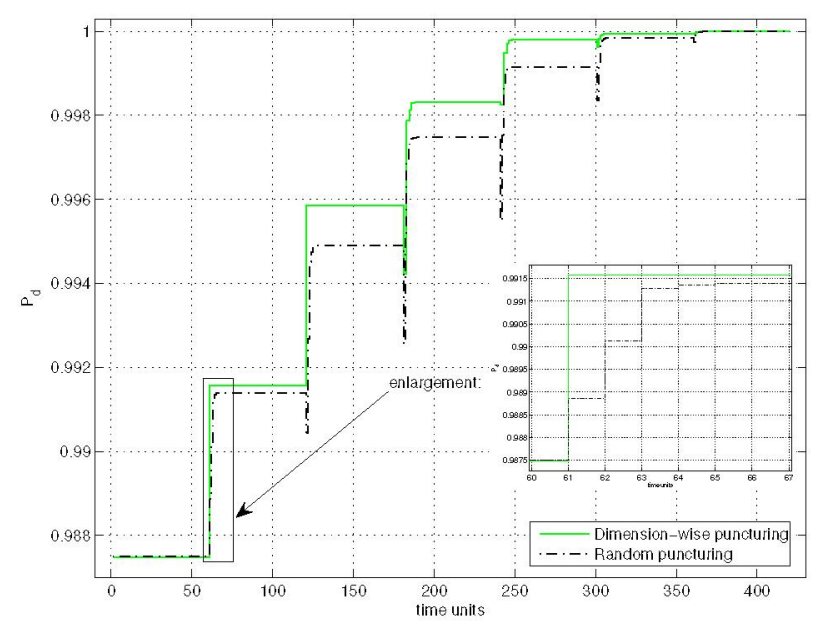

Figure 1. Comparison of two different puncturing patterns or transmission orders; random and dimension-wise, plotted at signal-to-noise ratio $4 \mathrm{~dB}$.

keying (BPSK) over an additive white Gaussian noise (AWGN) channel. To get an upper bound on performance, perfect error detection (PED) is used as the retransmission criterion and thus retransmissions will take place whenever a bit error occurs. Further, we assume an error free feedback channel, implying that no retransmission requests are ever lost or misinterpreted. In Figure 1 the QoS parameter $P_{d}$ is plotted as a function of time for the two different schemes. The time is given in time units rather than exact time since the latter would require knowledge of application specific parameters, such as distance between sender and receiver, transmission speed, decoder hardware architecture, etc. The round trip delay in this example is 60 time units and the first transmission arrives at time unit 0 . Hence, every 60 time unit additional parity bits will arrive if requested by the retransmission criterion.

The iterative decoder will initiate iterations as soon as parity bits belonging to two or more component decoders have been received. For the scheme using dimensionwise puncturing this does not occur until the fourth transmission has been received. For the scheme using random puncturing this may occur already in the second transmission arriving at time unit 60 . An activation of a component decoder is assumed to take one time unit in this example. Since the chosen mother code consists of three component codes, one iteration takes three time units since all component decoders have then been activated once. Looking at the dash-dotted curve in the enlargement figure within Figure 1, small steps can be noticed for time unit 61 to 67 corresponding to iterative refinements of $P_{d}$. Once one iteration has been completed at time unit 63, additional activations yield more and more diminishing returns.

Further, we can see that the scheme using random puncturing results in the value of $P_{d}$ being lowered at time unit 121, when iterations start after having received the third transmission. This is due to the fact that the iterative decoding process is restarted every time additional redundancy arrives. This implies that some information bits that were decoded correctly and thus improved $P_{d}$ are now temporarily reset when the iterative process is restarted. Since a retransmission occurs even if only one bit is in error, decoding is restarted even for data bits that were correctly decoded. This is why the level of $P_{d}$ drops at e.g. 121 time units. Note that the value of $P_{d}$ does not drop to the same level every time the decoding process is restated, since all packets need not be retransmitted.

From Figure 1 we can also see that dimension-wise puncturing is superior to random puncturing and thus also that the IR transmission order influences the performance. The better the puncturing pattern - the faster the level of $P_{d}$ increases and the earlier the correct result can be delivered.

The curves in Figure 1 at plotted at a signal-to-noise ratio of $4 \mathrm{~dB}$. At higher signal-noise ratios, the gain of dimension-wise puncturing is even higher [22]. Only for very high noise levels can random puncturing have a slight advantage. The performance of a particular puncturing pattern depends on the structure of the code. In this case, dimension-wise puncturing has an additional advantage in that only component decoders for which parity bits have been received need to be activated. This means that if only parity bits pertaining to one component encoder have been received no iterations are needed. This can be seen in Figure 1 where the solid curve lacks any small refinements until transmission four has been received at time unit 180 . When parity bits from two component decoders arrive, iterations include only these two decoders. This results in a less complex decoding procedure since one iterations entails only two activations.

The above results are based on an AWGN channel. In a wireless network, channel types such a block fading channels and other burst-error channels may be encountered. Concatenated coding is well-suited for such channels, as the parity bits in each dimension are based on encoding interleaved versions of the information bits. The decoding process therefore involves interleaved versions of information bits, allowing for some randomisation of burst errors. Further, the IR-HARQ scheme provides some inherent time diversity.

\section{IR packet sizes}

The truncated HARQ scheme considered here implies a limited number of retransmissions, which is controlled by the QoS parameters set by the real-time constraints. We assume that $t_{D L}$ together with knowledge of the bit transmission rate of the system will determine the maximum number of retransmissions allowed in order to get the required redundancy across in time. This means that while $P_{d}$ determines the necessary amount of redun- 
dancy, the bit transmission rate and $t_{D L}$ determines how many blocks the redundancy can be partitioned into, and hence also the maximum number of round-trip delays that can be afforded.

Given that we have a mother code that yields the required $P_{d}$ after the $M$ allowed retransmissions, we would like to limit the total amount of transmitted redundancy in order to use as little of the available resources as possible. This implies that we would like to maximize the code rate of the system. In the previous section it was concluded that a good puncturing pattern will lead to a higher $P_{d}$ earlier in time. This implies fewer retransmissions and a higher code rate. In this section we will show that by optimizing the amount of redundancy used in each transmission the code rate can be maximized, given a particular puncturing pattern.

The code rate of a non-ARQ system is $r_{C}=k / n$, but for an ARQ-system we can only get an average code rate since the retransmissions yields a variable code rate based on the current channel conditions. Assume that we have an IR-HARQ system that is limited to two packets, $M=2$, i.e., a packet of $n_{1}=k+c_{1}$ is transmitted first and thereafter a block of $c_{2}$ parity bits is transmitted only when necessary, resulting in a packet of length $n_{2}=k+c_{1}+c_{2}$. Consequently, when using this scheme, some of the frames have been accepted using a code rate of only $k /\left(k+c_{1}\right)$, whereas others needed a code rate of $k /\left(k+c_{1}+c_{2}\right)$. The average code rate of this IR-HARQ system is the mean of these two code rates. Since a code rate in some sense is a velocity, the harmonic mean must be used according to

$$
r_{C}^{M=2}=\left(a \frac{k+c_{1}}{k}+b \frac{k+c_{1}+c_{2}}{k}\right)^{-1},
$$

where $a$ and $b$ are the percentage of frames of the respective rate. We can express the average code rate as a function of the probability of a retransmission, $P_{A R Q}$, according to [23]

$$
r_{C}^{M=2}=\frac{k}{k+c_{1}+c_{2} P_{A R Q}\left(n_{1}\right)} .
$$

In a Monte-Carlo simulation, $P_{A R O}$ can be determined by counting the number of retransmissions actually made during the simulation. Note that the probability of a retransmission depends on the packet length $n_{1}$ in an IRHARQ system. Using PED yields $P_{A R Q}\left(n_{i}\right)$ equal to the frame error rate after decoding a codeword of length $n_{i}$. Generally, the more redundancy that is included in $n_{1}$ the smaller $P_{A R Q}\left(n_{1}\right)$ will be. Including more redundancy in the first transmission, however, also implies that the maximal average code rate is reduced. The expression in (2) can be generalize to $M$ number of transmissions as [23]

$$
r_{C}^{M}=\frac{k}{k+c_{1}+\sum_{i=2}^{M} c_{i} P_{A R Q}\left(n_{i-1}\right)} .
$$

The average code rate can be maximized by choosing optimal values on $c_{i}$ for $i=1,2, \ldots, M$. Recall that we have designed the mother code to cater for achieving the required level of $P_{d}$. This implies that in the last transmission, $c_{M}$ should always include all the remaining parity bits that have not yet been transmitted according to $c_{M}=n-k-\sum_{i=1}^{M-1} c_{i}$. For $M$ transmissions this results in an $M-1$ dimensional discrete maximization problem according to [23]

$$
\left[c_{1}^{*}, c_{2}^{*}, \ldots, c_{M-1}^{*}\right]=\arg \max _{c_{1}, c_{2}, \ldots c_{M-1}} r_{C}^{M}
$$

where $r_{C}^{M}$ is given by [23]

$$
r_{C}^{M}=\frac{k}{k+c_{1}+\sum_{i=2}^{M-1} c_{i} P_{A R Q}\left(n_{i-1}\right)+c_{M} P_{A R Q}\left(n_{M-1}\right)} .
$$

In Figure 2 a Monte-Carlo simulation of a scheme using the fixed packet sizes from Table 1 is compared to one using optimal packet sizes obtained analytically using (4). Both schemes use dimension-wise puncturing and hence the solid curve in Figure 1 is the same as the solid curve in Figure 2. The packet sizes for the two different schemes are given in Table 2.

Table 2. Packet sizes for the schemes used in Figure 2.

\begin{tabular}{|c|c|c|c|c|c|c|c|}
\hline & $n_{1}$ & $n_{2}$ & $n_{3}$ & $n_{4}$ & $n_{5}$ & $n_{6}$ & $n_{7}$ \\
\hline Fixed: & 343 & 367 & 392 & 416 & 441 & 465 & 490 \\
\hline Optimal: & 422 & 427 & 433 & 441 & 457 & 473 & 490 \\
\hline
\end{tabular}

The scheme using fixed packet sizes has received all of the parity bits from the first dimension after three transmissions and all parity bits from the second dimension after five transmissions. The scheme using optimal packet sizes have received all the parity bits from the first dimension and also some parity bits from the second dimension already in the very first transmission. Hence, the scheme with optimal packet lengths will start iterating immediately after the first transmission. Again it is assumed that a new transmission arrives every 60 time unit if the retransmission criterion requires additional parity bits and that one activation of a component decoder takes one time unit.

It can be seen that using optimal packet sizes yields significant performance improvements in terms of 


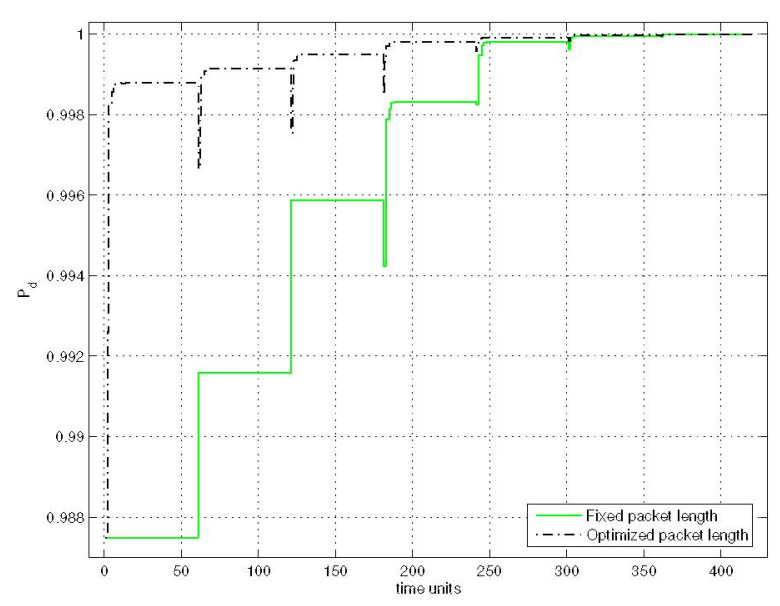

Figure 2. Comparison of IR schemes using fixed packet lengths and optimized packet lengths. Plotted at signal-to-noise ratio $4 \mathrm{~dB}$.

maximizing the average code rate. This implies that we us less redundancy and therefore less resources, but also that the average communication time is reduced. Using all seven retransmission corresponds to the worst case since each retransmission increases the probability that the frame is correctly decoded and further retransmissions becomes unnecessary

The curves in Figure 2 are again plotted at a signal-tonoise ratio of $4 \mathrm{~dB}$. A higher signal-noise ratio results in a different set of optimal packet lengths as reported in Table 3. As can be seen less and less redundancy is required in the first transmission as the signal-to-noise ratio increases. If simple transmitters and receivers are used, the optimization of packet lengths may be done using a look-up table as in Table 3 . However, if more costly receivers are available the choice of packet lengths can be done adaptively based on the current estimated channel conditions.

Table 3. Optimal packet lengths for different signal-to-noise ratios in $\mathrm{dB}$.

\begin{tabular}{|c|c|c|c|c|c|c|c|}
\hline$[\mathrm{dB}]$ & $n_{1}$ & $n_{2}$ & $n_{3}$ & $n_{4}$ & $n_{5}$ & $n_{6}$ & $n_{7}$ \\
\hline 2.6 & 457 & 460 & 464 & 469 & 475 & 482 & 490 \\
\hline 4.0 & 422 & 427 & 433 & 441 & 457 & 473 & 490 \\
\hline 5.3 & 387 & 392 & 405 & 416 & 427 & 441 & 490 \\
\hline 6.6 & 343 & 364 & 379 & 392 & 426 & 441 & 490 \\
\hline 8.0 & 343 & 369 & 381 & 392 & 467 & 478 & 490 \\
\hline 9.0 & 343 & 374 & 384 & 392 & 424 & 441 & 490 \\
\hline 10.0 & 343 & 378 & 386 & 392 & 469 & 479 & 490 \\
\hline 11.0 & 343 & 380 & 383 & 387 & 390 & 392 & 490 \\
\hline
\end{tabular}

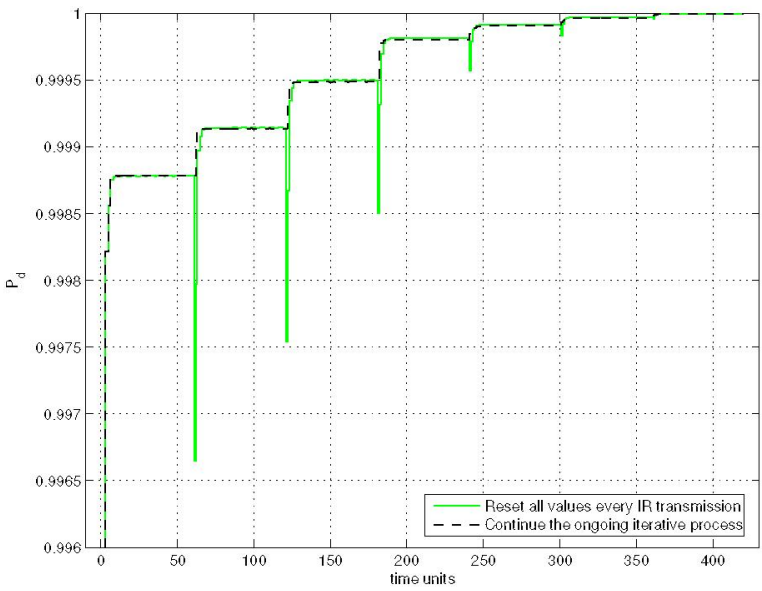

Figure 3. A scheme not restarting the iterative decoding process each retransmission is compared to one that does. Plotted at signal-to-noise ratio $4 \mathrm{~dB}$.

A scheme that does not restart the iterative decoding process each time additional parity bits arrive will gain even further time. In Figure 3 two schemes using dimension-wise puncturing and optimal packet lengths are plotted. One restarts the iterative process each time a new transmission arrives (the solid curve in Figure 3, which is also the same as the dash-dotted curve in Figure 2 ), whereas the other does not (the dashed curve in Figure 3 ). The scheme that does not restart the iterative process requires fewer iterations to converge. If an iteration is very complex or energy demanding, this procedure may be advantageous.

\section{Conclusions}

The DDC scheme using IR evaluated here constitutes a flexible and robust communication scheme that carefully manages the available resources while keeping the real-time constraints. The QoS parameters deadline $t_{D L}$ and probability of correct delivery prior to the deadline $P_{d}$ determines the required redundancy and the maximum number of retransmissions allowed. An admission control system using this protocol is able to provide a trade-off between the worst case delivery time and the quality of the delivered data. Service requests can therefore be accepted, rejected or renegotiated depending on the available resources. The retransmission scheme enables early termination of the communication, as soon as the required quality has been obtained. Thereby the use of available resources is kept at a minimum. Using powerful concatenated codes with iterative decoding enables improved fault-tolerance over an unreliable wireless channel. It is shown that by choosing an appropriate transmission order and optimal partition of the redundancy, the required level of $P_{d}$ can be obtained at an even earlier point in time. 


\section{References}

[1] J. G. Proakis, Digital Communications, 3rd ed., McGraw-Hill, New York, NY, 1995.

[2] H. Bengtsson, E. Uhlemann, and P.-A. Wiberg, "Protocol for wireless real-time systems," in Proc. Euromicro Conference on Real-Time Systems, York, UK, June 1999, pp. 168-174.

[3] E. Uhlemann, T. M. Aulin, L. K. Rasmussen, and P.-A. Wiberg, "Deadline dependent coding - a framework for wireless real-time communication," in Proc. International Conference on Real-Time Computing Systems and Applications, Cheju Island, South Korea, December 2000, pp. 135-142.

[4] E. Uhlemann, T. M. Aulin, L. K. Rasmussen, and P.-A. Wiberg, "Concatenated hybrid ARQ - a flexible scheme for wireless real-time communication," in Proc. IEEE Real-Time Embedded Technology and Applications Symposium, San Jose, CA, September 2002, pp. 35-44.

[5] C. Berrou, A. Glavieux, and P. Thitimajshima, "Near Shannon limit error-correcting coding and decoding: turbo codes," in Proc. International Conference on Communications, Geneva, Switzerland, May 1993, pp. 1064-1070.

[6] D. M. Mandelbaum, "An adaptive-feedback coding scheme using incremental redundancy," IEEE Transactions on Information Theory, vol. 20, no. 3, pp. 388-389, May 1974.

[7] J. F. Kurose and K. W. Ross, Computer Networking - A Top-Down Approach Featuring the Internet, AddisonWesley, 2000.

[8] A. Leon-Garcia and I. Widjaja, Communication Networks: Fundamental Concepts and Key Architectures, McGraw-Hill, 2000.

[9] W. Zhao, J. A. Stankovic, and K. Ramamritham, "A window protocol for transmission of time-constrained messages," IEEE Transactions on Computers, vol. 39, no. 9, pp. 1186-1203, September 1990.

[10] S.-K. Kweon, K. G. Shin, and Q. Zheng, "Statistical real-time communication over Ethernet for manufacturing automation systems," in Proc. IEEE Real-Time Technology and Applications Symposium, Phoenix, AZ, May 1999, pp. 192-202.

[11] A. G. Argawal, B. Chen, W. Zhao, and S. Davari, "Guaranteeing synchronous message deadlines with the timed token medium access control protocol," IEEE Transactions on Computers, vol. 43, no. 3, pp. 327-339, March 1994.
[12] T. F. Abdelzaher, E. M. Atkins, and K. G. Shin, "QoS negotiation in real-time systems and its application to automated flight control," IEEE Transactions on Computers, vol. 49, no. 11, pp. 1170-1183, November 2000.

[13] J. Kim and K. G. Shin, "Performance evaluation of dependable real-time communication with elastic QoS," in Proc. International Conference on Dependable Systems and Networks, Gothenburg, Sweden, July 2001, pp. 295303.

[14] C. E. Shannon, "A mathematical theory of communication," Bell System Technical Journal, vol. 27, pp. 379423 and pp. 623-656, October 1948.

[15] G. D. Forney, Jr., Concatenated Codes, M.I.T. Press, Cambridge, MA, 1966.

[16] S. Lin, D. J. Costello, Jr., and M. J. Miller, "Automaticrepeat-request error control schemes," IEEE Communications Magazine, vol. 22, no. 12, pp. 5-16, December 1984.

[17] J. M. Wozencraft and M. Horstein, "Digitalised communication over two-way channels," in Proc. Fourth London Symposium on Information Theory, London, U.K., September 1960.

[18] P. Sindhu, "Retransmission error control with memory," IEEE Transactions on Communications, vol. 25, no. 5, pp. 423-429, May 1977.

[19] S. Lin and P. S. Yu, "A hybrid ARQ scheme with parity retransmission for error control of satellite channels," IEEE Transactions on Communications, vol. 30, no. 7 , pp. 1701-1719, July 1982.

[20] D. Chase, "Code combining - a maximum-likelihood decoding approach for combining an arbitrary number of noisy packets," IEEE Transactions on Communications, vol. 33, no. 5, pp. 385-393, May 1985.

[21] S. Kallel, "Complementary punctured convolutional (CPC) codes and their applications," IEEE Transactions on Communications, vol. 43, no. 6, pp. 2005-2009, June 1995.

[22] E. Uhlemann, Adaptive Concatenated Coding for Wireless Real-Time Communications, Ph.D. thesis, Chalmers University of Technology, Göteborg, Sweden, September 2004.

[23] E. Uhlemann, L. K. Rasmussen, A. J. Grant, and P.-A. Wiberg, "Optimal incremental-redundancy strategy for type-II hybrid ARQ," in Proc. IEEE International Symposium on Information Theory, Yokohama, Japan, June 2003 , pp. 448. 